\title{
Situación de la confianza en la teoría fundamentada versus la modelación de ecuaciones estructurales: un estudio bibliométrico comparativo para la administración
}

\section{Pablo José Arana Barbier*}

Artículo recibido:

25 de abril de 2019

Artículo aceptado:

16 de agosto de 2019

Artículo de investigación

\section{Resumen}

Los paradigmas cuantitativo y cualitativo son utilizados por millones de investigadores, pero no se conoce el nivel de confianza depositado en cada uno de ellos. El objetivo del artículo bibliométrico es estudiar a la teoría fundamentada versus la modelación de ecuaciones estructurales para determinar en cuál de las dos metodologías confía más la comunidad científica. A través de estadística descriptiva y regresional, los hallazgos más relevantes consisten en que, si bien la comunidad científica confía ligeramente más en el paradigma cuantitativo que en el cualitativo, esta situación tendería a revertirse para 2022, dando un giro hacia el cualitativo.

* Centrum Católica Graduate Business School (CCGBS), Pontificia Universidad Católica del Perú (PUCP)

pablo.arana@pucp.pe

INVESTIGACIÓN BIBLIOTECOLÓGICA, vol.34, núm. 83, abril/junio, 2020, México, ISSN: 2448-8321 pp. 37-53 
Palabras clave: Citaciones por Artículo; Confianza; Modelación de Ecuaciones Estructurales; Teoría Fundamentada

\author{
Situation of trust in grounded theory versus struc- \\ tural equation modeling: a comparative bibliometric \\ study for management \\ Pablo José Arana Barbier
}

\begin{abstract}
The quantitative and qualitative paradigms are used by millions of researchers, but the level of confidence deposited in each one of them it is not known. The aim of the bibliometric article is to study the grounded theory versus the structural equations modeling in order to determine in which of the two methodologies the scientific community relies the most. Through descriptive and regression statistics, the most relevant findings are that, although the scientific community relies slightly more on the quantitative than on the qualitative paradigm, this situation would tend to revert by 2022 , taking a turn towards the qualitative one.
\end{abstract}

Keywords: Citations Per Paper; Grounded Theory; Trust; Structural Equation Modeling

\title{
INTRODUCCIÓN
}

$\mathrm{L}$ a teoría fundamentada o grounded theory (GT) revolucionó las metodologías de investigación (Glaser y Strauss, 1967; Sutcliffe, 2016). No obstante, ha recibido tanto elogios (Burton, 2007; Stern, 2007) como cuestionamientos (Blalock, 1970; Bryman, 2004; Cutcliffe, 2000). Urquhart y Fernández (2013) resumieron los últimos en tres: a) el investigador no revisa previamente literatura; b) la GT es inflexible, y c) produce conocimiento de bajo nivel. Sin embargo, la revisión previa de literatura fue incluida como parte de la metodología actualizada (Strauss y Corbin, 1990), se probó la flexibilidad de la teoría (Tan, 2010) por su enfoque constructivista (Charmaz, 
2000), y se propuso que las entrevistas a profundidad son suficientes, en reemplazo de una escala de Likert, para generar conocimiento (Graue, 2015; Holland, 1998; Pinegar y Wilbricht, 1989).

Es decir, existe evidencia suficiente para afirmar que la GT es una herramienta potente para generar conocimiento (Jones, 2009). Gioia, Corley y Hamilton (2013) señalaron que la GT es rigurosa desde el planteamiento de las proposiciones, pasando por la codificación, hasta la interpretación de la información. Creswell (2007) la consideró dentro de los cinco principales métodos de investigación cualitativa. Sin embargo, ¿podría la metodología cualitativa, en particular la GT, ocupar un rol más relevante en la generación de conocimiento vinculado a la administración?

La postura de trabajar lo financiero a través del paradigma cualitativo ya ha sido defendida (Burton, 2007). Además, existen investigaciones (Khresna, Hooy y Ahmad, 2012) que plantean que las decisiones humanas tienden a ser irracionales, incluidas las financieras (Viktorovna, Pavlovna y Mokhailovna, 2018), y no sólo a nivel de individuos, sino de mercados también (Poteshman y Serbin, 2003). Desde luego, ello no involucra que toda decisión humana sea irracional, pero los argumentos previos en conjunto soportarían la postura de Graue (2015) de estudiar al individuo que toma la decisión, y no a la decisión misma. Sin embargo, no sólo cuenta el esfuerzo de desarrollar ciencia a través del paradigma cualitativo, sino en qué medida los investigadores confían en que dichos resultados son insumos veraces para continuar construyendo conocimiento. De acuerdo con Nicolson (2013), un hecho se considera verdad por el respaldo que recibe de los individuos que componen la comunidad que acepta dicha verdad. Si bien existen métodos para probar la validez (Yin, 2003; Charmaz, 2006) y confiabilidad (Long y Johnson, 2000; Bengtsson, 2016) de la investigación cualitativa, ello no necesariamente involucra que la comunidad científica confiará en sus resultados, los considerará una verdad (Nicolson, 2013) y los utilizará como insumo para sus propias investigaciones.

Resulta relevante, además, comprender el término confianza. Giffin (1967) la definió como la "dependencia sobre las características de un objeto, o la ocurrencia de un evento, o el comportamiento de una persona para alcanzar un deseado pero incierto objetivo en una situación riesgosa" (Giffin, 1967: 105). Por otra parte, Cronin (1984) señaló que un científico recibe el reconocimiento público de sus pares al publicar su investigación, mientras que al mismo tiempo, a quienes dicho científico citó, reciben el reconocimiento por el rol que cumplieron dentro de su investigación. Así, al combinar lo propuesto por a) Giffin (1967), b) Cronin (1984) y c) Nicolson (2013), en el artículo la confianza en una investigación girará en torno a si la comunidad científica la considera como un insumo valioso, y si está dispuesta a depender de ella como sustento para la generación de nuevo conocimiento. 
Respecto al proceso de revisión y publicación de una investigación, asumiendo que la revisión de los árbitros obedeció a consideraciones de objetividad, rigurosidad y ética (Berk, Harvey y Hirshleifer, 2017), el artículo podría ser aceptado para publicación. No obstante, la comunidad científica no está obligada a citarlo. Esto ocurrirá sólo si le resulta útil y si confía en él (Cronin, 1984; Giffin, 1967; Nicolson, 2013), confianza que tendría que traducirse en una citación para continuar construyendo conocimiento (Cronin, 1984). La literatura actual carece de investigaciones que estudien el comportamiento de la confianza en la metodología cualitativa. Existe un punto de partida en el que la comunidad científica dedicó un esfuerzo considerable para criticar a la GT (Brown, 1973; Bryman, 2004; Cutcliffe, 2000), pero no un registro de la evolución progresiva de la crítica pasada hasta la defensa actual sustentado en el volumen de publicaciones realizadas y citaciones recibidas. Por ello es que existe una excelente oportunidad de estudiar su evolución y situación actual.

La literatura bibliométrica especializada (Moed et al., 1985; Verbeek et al., 2002) también ofrece una oportunidad de investigación, ya que considera el índice de citaciones por artículo como un indicador de impacto (Candia et al., 2019), y no de confianza (Cronin, 1984; Giffin, 1967; Nicolson, 2013). Además, muchos estudios bibliométricos segmentan sus hallazgos de diferentes formas (Cobo et al., 2015; Merigó et al., 2015; Merigó y Yang, 2017), pero no proponen una comparación entre paradigmas cuantitativos o cualitativos. Estos vacíos ofrecen una oportunidad para investigar si la comunidad científica confía en mayor o menor medida en el paradigma cualitativo versus el cuantitativo.

Factores varios también pueden influir sobre la citación de un artículo. Por ejemplo, a) el prestigio de los autores, b) el número de citaciones recibidas o c) el prestigio de la revista (Thornley et al., 2015). Por otro lado, Di Vaio, Waldenström y Weisdorf (2012) hallaron que existe una mayor probabilidad de ser citado cuando a) se participe en seminarios o conferencias mediante trabajos en curso, b) si las investigaciones son producidas en coautoría, o c) si es que los autores son profesores a tiempo completo. Simkin y Roychowdhury (2003) señalaron que sólo 20 \% de las referencias en una investigación realmente fueron leídas por los autores. Además, Cintra, Furnival y Milanez (2018) indicaron que el acceso abierto a las revistas también influye positivamente en el número de citaciones que recibe una investigación.

Otros fenómenos bibliométricos denotan claramente que, dependiendo también de las disciplinas estudiadas, puede existir mayor preferencia por una metodología en particular. Por ejemplo, Strandberg et al. (2018) detectaron que en la industria del turismo ha habido una importante migración 
hacia la metodología cuantitativa en los últimos 15 años. Por otro lado, Cornelius y Persson (2006) hallaron que los investigadores de finanzas y economía tienden más hacia lo cuantitativo, mientras que los de gestión y emprendimiento prefieren lo cualitativo. Desde luego, dichos estudios no pueden ser generalizados, y junto con los claros resultados opuestos, ofrecen una oportunidad para profundizar en la situación actual de ambas metodologías y qué tan preferidas son entre los investigadores.

El objetivo del artículo, por lo tanto, es analizar el comportamiento de la citación de los artículos cualitativos versus cuantitativos como un reflejo de la confianza (Cronin, 1984; Giffin, 1967; Nicolson, 2013) en ambos métodos para los campos de economics, management y business, los más relevantes según Liu, Hong y Liu (2012). Se prestará particular atención a la metodología más popular de cada enfoque, a través de la estadística descriptiva de cantidad de publicaciones y citaciones en un horizonte de 15 años concluidos (2004-2018), para finalmente comparar los patrones de crecimiento de las citaciones a través del análisis regresional.

$\mathrm{El}$ aporte de la investigación es significativo principalmente por tres motivos. Primero, la evolución a través del tiempo de la producción científica basada en GT no se ha documentado, y por lo tanto no se conoce si su desarrollo ha sido acompañado por producción intelectual o mayor número de citaciones. Segundo, la literatura relevante sobre la comparación de la confianza depositada en los paradigmas cuantitativo y cualitativo traducida en citaciones por artículo es incipiente (Cronin, 1984; Giffin, 1967; Nicolson, 2013). Tercero, la investigación inicia la discusión y análisis de las citaciones por artículo ya no como un indicador de impacto, sino de confianza (Cronin, 1984; Giffin, 1967; Nicolson, 2013).

\section{Metodología}

El estudio consideró los resultados de búsquedas en Scopus de dos metodologías de investigación en artículos publicados en revistas durante 15 años, de 2004 a 2018. Las revistas correspondieron únicamente a las categorías "Business, Management and Accounting", y "Economics, Econometrics and Finance", tal y como figuran en el sistema de búsqueda de Scopus y vinculadas a los campos de estudio de economics, management y business (Liu, Hong y Liu, 2012), los cuales sirvieron para delimitar los documentos a ser analizados, mas no representaron factores a analizar respecto a campo de conocimiento, contexto de origen de las publicaciones o interés de las mismas. La metodología cualitativa escogida es la principalmente defendida por Creswell 
(2007): la GT, ingresada al buscador de Scopus como "grounded theory". La metodología cuantitativa es la más defendida por Chión y Charles (2016): la modelación de ecuaciones estructurales (SEM), ingresada al buscador de Scopus como "structural equation modeling". Un punto importante para comparar dichas metodologías radica en que, además de ser la más relevante de acuerdo a Chión y Charles (2016), ambas fueron formalmente propuestas en la misma época: la SEM se desarrolló desde inicios del siglo XX hasta su proposición formal por K. Jöreskog en 1970 (Mateos-Aparicio, 2011), versus la propuesta de la GT por parte de Glaser y Strauss (1967). Si bien su horizonte temporal promueve su comparación, resultará relevante también para futuras investigaciones utilizar otras metodologías cuantitativas para efectos de comparación.

La búsqueda de los términos en inglés se realizó entre los días 10 y 11 de abril de 2019 mediante la opción Article title, abstract and keywords en el buscador inicial de la base Scopus. La búsqueda se delimitó por a) año (20042018), b) tipo de documento (articles), y c) tipo de fuente (journals). Ninguno de ellos se encuentra en etapa de prensa. La búsqueda arrojó cuántos artículos se publicaron cada año, además de la cantidad de citaciones por año de cada artículo hallado, para obtener la razón de citaciones promedio por artículo por año. El análisis estadístico de las citaciones promedio es considerado relevante para comprender el comportamiento de la generación de conocimiento (Martin, 2012; Zupic y Čater, 2015), y sustentado por diversos autores (Cobo et al., 2015; Merigó et al., 2015; Merigó y Yang, 2017; Moed et al., 1985; Verbeek et al., 2002).

Si bien no se ha documentado la evolución de la situación de confianza en la GT ni se ha comparado contra una metodología cuantitativa, ha sido respaldada por muchos autores. Por ello, la hipótesis 1 es la siguiente:

$\mathrm{H}_{1}$ : la confianza actual medida en citaciones promedio por artículo es igual para artículos de SEM que para artículos de GT.

Basado en ese mismo razonamiento, si la GT continuara demostrando su validez y la confianza en ella se incrementara, se esperaría la hipótesis 2:

$\mathrm{H}_{2}$ : la confianza futura medida en citaciones promedio por artículo para artículos de SEM será menor que para artículos de GT.

Posterior a la extracción de la información, mediante las citaciones promedio se llevó a cabo el análisis descriptivo y regresional para analizar la tendencia 
de comportamiento de las mismas. La información extraída se puede clasificar en dos: a) número de artículos, definido como la cantidad de investigaciones publicados en un año, y b) citaciones por año, que representa la cantidad de investigaciones que han citado a los artículos estudiados en cada uno de los años analizados. Para las citaciones promedio por año no se consideró el número neto de publicaciones por año, sino el acumulado de publicaciones a la fecha desde el primer año estudiado, ya que los artículos pasados continúan aportando a las citaciones a través del tiempo.

\section{Presentación y anÁlisis De Resultados}

La Tabla 1 muestra los resultados de búsqueda de structural equation modeling (SEM), mientras que la Tabla 2 lista los correspondientes a grounded theory (GT). Los artículos de SEM publicados en 2004 fueron 93, y 1955 en 2018. El total de publicaciones de SEM para los 15 años estudiados fue de 10 972, aunque cabe resaltar que 2429 de ellas (22.14 \%) no presentaron citación alguna, 8519 (77.64\%) tuvieron entre 1 y 500 citaciones en total, y 24 $(0.22 \%)$ excedieron las 500 citaciones totales. Respecto a la tasa de citaciones promedio por artículo, creció de 0.10 en 2004 a 4.80 en 2018.

Respecto a los artículos de GT, ascendieron en 2004 a 40, mientras que en 2018 fueron 189. El total de investigaciones publicadas en los 15 años estudiados fue de 1616 , de las cuales 312 (19.31\%, versus $22.14 \%$ de SEM) no presentaron citaciones, 1301 tuvieron entre 1 y 500 citaciones ( $80.51 \%$, versus $77.64 \%$ de SEM), y sólo 3 ( $0.19 \%$, versus $0.22 \%$ de SEM) excedieron las 500 citaciones totales. La tasa de citaciones promedio por artículo pasó de 1.43 en 2004 a 3.61 en 2018. Las cantidades son muy similares en proporción a las presentadas por los artículos de SEM.

El comportamiento gráfico de las citaciones se encuentra resumido en la Figura 1, la cual presenta las siguientes vistas: a) artículos totales por metodología estudiada, b) citaciones totales por metodología estudiada, c) citaciones promedio por metodología estudiada, y d) citaciones promedio por metodología estudiada, incluidas las regresiones lineales y polinómicas de segundo grado. En el cuadrante superior izquierdo se muestra el comportamiento exponencial de la producción intelectual publicada de SEM, frente al comportamiento lineal de producción intelectual de GT. Algo muy similar ocurre en el cuadrante superior derecho, con un crecimiento exponencial de citaciones de artículos de SEM versus el crecimiento lineal de citaciones de investigaciones de GT. No obstante, la tendencia cambia en el cuadrante inferior izquierdo, ya que no se evidencian crecimientos exponenciales. Durante los primeros tres años analizados, las citaciones promedio por artículo son mayores para investigaciones de GT, pero dicha tendencia se revierte en 2007 y se mantiene hasta 2018. 


\begin{tabular}{|c|c|c|c|c|c|c|c|c|c|c|}
\hline$\stackrel{\infty}{\check{N}}$ & 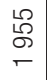 & $\begin{array}{l}\text { Ñ } \\
\text { Oे } \\
\stackrel{0}{0}\end{array}$ & $\begin{array}{l}\overline{\tilde{m}} \\
\mathbb{0} \\
\tilde{N}\end{array}$ & $\begin{array}{l}\infty \\
\infty \\
\forall\end{array}$ & \multirow{16}{*}{ 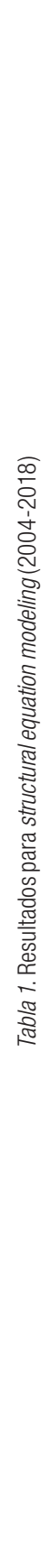 } & $\stackrel{\infty}{\check{\Sigma}}$ & 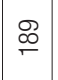 & $\underset{\bullet}{\stackrel{0}{6}}$ & $\begin{array}{l}\infty \\
\infty \\
\infty \\
\infty \\
\infty\end{array}$ & $\bar{c}$ \\
\hline 令 & $\begin{array}{l}5 \\
\stackrel{\circ}{\circ} \\
-\end{array}$ & స్ & $\begin{array}{l}\text { ले } \\
\text { స్m }\end{array}$ & $\underset{\forall}{\stackrel{\sim}{\sim}}$ & & $\stackrel{\sim}{\circ}$ & $\stackrel{\mathscr{m}}{\stackrel{\rho}{\sim}}$ & $\underset{\leftarrow}{\stackrel{\Im}{\sim}}$ & \begin{tabular}{l}
$\infty$ \\
\multirow{2}{0}{} \\
$\forall$
\end{tabular} & $\begin{array}{l}\stackrel{\infty}{\infty} \\
\sim\end{array}$ \\
\hline$\stackrel{0}{\circ}$ & $\begin{array}{c}\underset{\infty}{\infty} \\
\underset{\leftarrow}{+}\end{array}$ & $\underset{\sim}{\stackrel{N}{\sim}}$ & 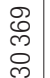 & $\underset{\sim}{\stackrel{g}{*}}$ & & $\stackrel{\circ}{\stackrel{0}{N}}$ & $\begin{array}{l}\infty \\
\stackrel{\infty}{\leftarrow} \\
\end{array}$ & $\underset{\sim}{\stackrel{N}{\sim}}$ & 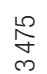 & 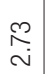 \\
\hline$\stackrel{10}{2}$ & $\underset{\leftarrow}{\stackrel{f}{ \pm}}$ & $\begin{array}{l}\stackrel{J}{\Delta} \\
\stackrel{5}{\circ}\end{array}$ & $\begin{array}{l}\llcorner \\
0 \\
0 \\
\infty \\
\sim\end{array}$ & $\begin{array}{l}\infty \\
\stackrel{\infty}{\infty} \\
\end{array}$ & & $\stackrel{\circ}{\frac{1}{2}}$ & $\underset{\widetilde{N}}{\mathbb{N}}$ & \begin{tabular}{l}
$\stackrel{8}{0}$ \\
0 \\
\hdashline
\end{tabular} & $\begin{array}{l}\text { f } \\
\infty \\
\sim\end{array}$ & $\bar{\sim}$ \\
\hline$\stackrel{\nabla}{\stackrel{D}{\sim}}$ & 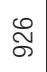 & $\underset{\checkmark}{\stackrel{D}{\nabla}}$ & 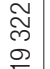 & $\underset{\forall}{\mathscr{\forall}}$ & & $\stackrel{+}{\circ}$ & $\stackrel{\mathbb{N}}{\mathbb{N}}$ & $\widehat{\widehat{o}}$ & $\stackrel{\infty}{\underset{\sim}{\sim}}$ & $\stackrel{\widehat{m}}{\sim}$ \\
\hline$\stackrel{\text { m. }}{\stackrel{\sim}{\sim}}$ & $\frac{O}{R}$ & $\begin{array}{l}\bar{T} \\
\infty \\
\infty\end{array}$ & \begin{tabular}{l}
\multirow{J}{*}{} \\
$\infty$ \\
$\forall$ \\
$\forall$
\end{tabular} & 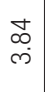 & & $\stackrel{m}{\stackrel{n}{\sim}}$ & $\stackrel{\infty}{\simeq}$ & $\begin{array}{l}\infty \\
\infty\end{array}$ & $\begin{array}{l}\stackrel{0}{2} \\
0 \\
\leftarrow\end{array}$ & $\underset{\sim}{\sim}$ \\
\hline ָั & $\begin{array}{l}8 \\
\& \\
0\end{array}$ & $\frac{\widetilde{N}}{\infty}$ & $\begin{array}{l}\stackrel{̊}{0} \\
\stackrel{\circ}{0} \\
\mp\end{array}$ & $\begin{array}{l}\hat{\oplus} \\
\dot{m}\end{array}$ & & $\stackrel{\sim}{\check{\sim}}$ & के & $\begin{array}{l}: \\
: 0 \\
0\end{array}$ & $\underset{\text { 守 }}{-}$ & $\stackrel{\circ}{\sim}$ \\
\hline Б) & $\underset{\omega}{\approx}$ & $\underset{\sim}{\stackrel{\sim}{\sim}}$ & 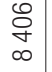 & $\begin{array}{l}\stackrel{+}{+} \\
\text { ஸे }\end{array}$ & & 产 & 응 & $\begin{array}{l}\mathscr{M} \\
\infty \\
\infty\end{array}$ & $\underset{\widetilde{N}}{\stackrel{\widetilde{N}}{\sim}}$ & $\stackrel{\oplus}{\stackrel{\sim}{\sim}}$ \\
\hline 웅 & $\begin{array}{l}\infty \\
\stackrel{9}{+}\end{array}$ & 요 & $\begin{array}{l}\hat{} \\
\infty \\
0\end{array}$ & $\begin{array}{l}\infty \\
\circ \\
\dot{m}\end{array}$ & & 윰 & $\stackrel{\checkmark}{\circ}$ & $\stackrel{\mathscr{O}}{\stackrel{\infty}{+}}$ & ఠ̊ & $\stackrel{\stackrel{g}{\circ}}{\stackrel{\circ}{\leftarrow}}$ \\
\hline ஓे & 마 & ণ্ণ & $\begin{array}{l}0 \\
\text { ల } \\
\stackrel{\sigma}{0}\end{array}$ & $\begin{array}{l}\infty \\
\infty \\
\sim \\
\sim\end{array}$ & & ஓे & के & 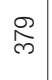 & $\stackrel{8}{2}$ & $\begin{array}{l}\infty \\
\infty \\
-\end{array}$ \\
\hline$\stackrel{\infty}{\circ}$ & $\stackrel{\varphi}{m}$ & $\overline{8}$ & 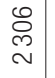 & $\begin{array}{l}\stackrel{p}{\sim} \\
\stackrel{\sim}{ }\end{array}$ & & $\stackrel{\infty}{\stackrel{\overbrace{}}{े}}$ & $\Re$ & $\stackrel{\sim}{\sim}$ & $\stackrel{g}{\stackrel{9}{\sigma}}$ & $\stackrel{?}{\stackrel{P}{\leftarrow}}$ \\
\hline ঠे & $\stackrel{\circ}{\stackrel{\circ}{N}}$ & $\begin{array}{l}\llcorner \\
\stackrel{0}{0} \\
0\end{array}$ & 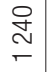 & $\stackrel{\bar{\infty}}{-}$ & & ¿̊․ & L & $\stackrel{\sim}{\stackrel{\circ}{2}}$ & 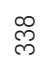 & $\underset{\widetilde{\Xi}}{\tilde{\Xi}}$ \\
\hline $\begin{array}{l}\stackrel{\circ}{\circ} \\
\text { ㅇ }\end{array}$ & के & 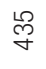 & $\begin{array}{l}\text { L } \\
\text { م }\end{array}$ & $\stackrel{\widehat{m}}{\leftarrow}$ & & $\begin{array}{l}\text { ஜ } \\
\text { ¿ }\end{array}$ & $\begin{array}{ll}\infty \\
\end{array}$ & $\underset{\square}{\stackrel{J}{\Xi}}$ & $\stackrel{\circledast}{\curvearrowright}$ & 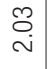 \\
\hline 용 & 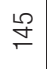 & $\stackrel{\infty}{\sim}$ & $\underset{\widetilde{O}}{\widetilde{0}}$ & $\begin{array}{l}\infty \\
\stackrel{0}{0} \\
\stackrel{\leftrightarrow}{0}\end{array}$ & & $\stackrel{\llcorner}{\circ}$ & 文 & $\stackrel{\infty}{\infty}$ & ঙ্ণ & 怘 \\
\hline ঠి & Sె & חু & $\sigma$ & $\frac{}{\circ}$ & & ষ্ণ & P & P & 㘦 & $\stackrel{\mathscr{P}}{\leftarrow}$ \\
\hline 岕 & 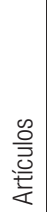 & 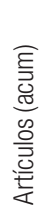 & 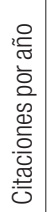 & 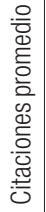 & & 心 & 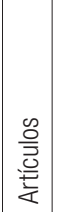 & 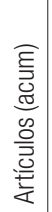 & 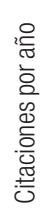 & 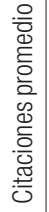 \\
\hline
\end{tabular}




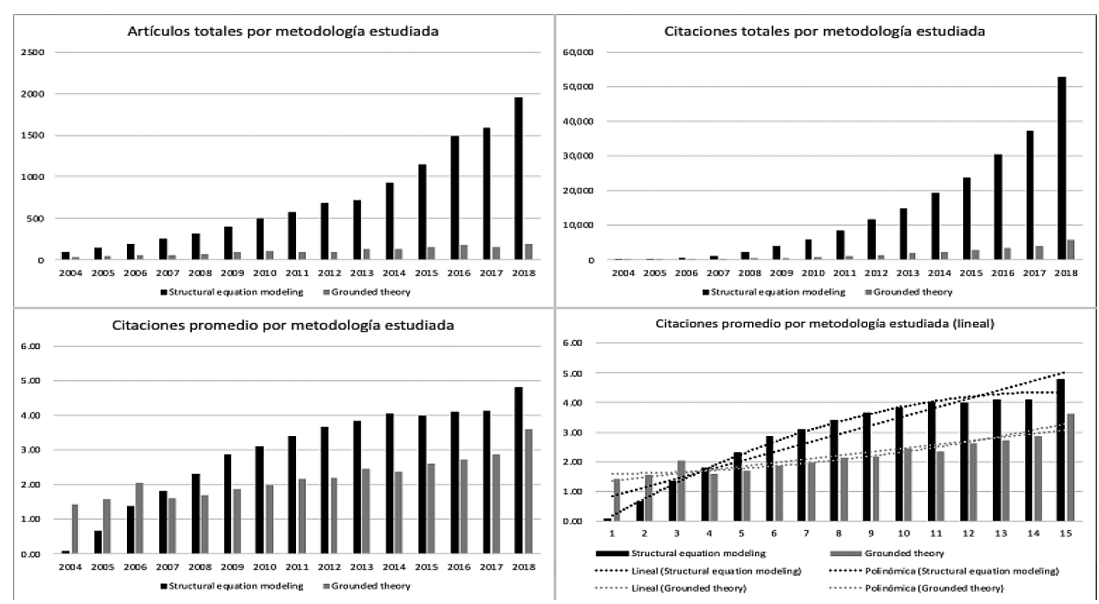

Figura 1. Comportamiento gráfico del total de artículos y citaciones promedio por artículo para las dos metodologías estudiadas

El cuadrante inferior derecho de la Figura 1 analiza las tendencias regresionales, tanto lineales como polinómicas de grado 2. A través de Microsoft Excel se plantearon las rectas de mejor ajuste lineales para los gráficos de barras, considerando 2004 como el período 1 y 2018 como el período 15. A continuación se muestra el resultado para las citaciones promedio de SEM:

$$
y=0.2982 x+0.558
$$

La regresión arrojó un coeficiente $\mathrm{R}^{2}$ de 0.9104, mayor que 0.70 (Véliz, 2011). La Figura 2 muestra sus pruebas estadísticas. Para ambos coeficientes, los p-values están por debajo de 0.05 , y ninguno de los intervalos de confianza pasa por 0 .

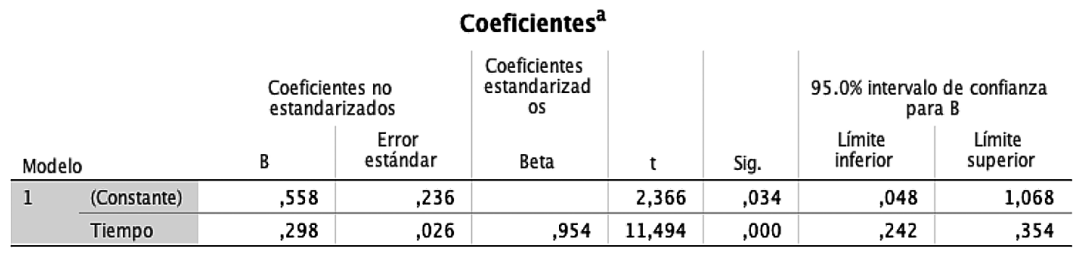

a. Variable dependiente: CitproSEM

Figura 2. Análisis estadístico en SPSS para citaciones promedio de SEM (regresión lineal) 
La expresión lineal para las citaciones promedio de GT se muestra a continuación:

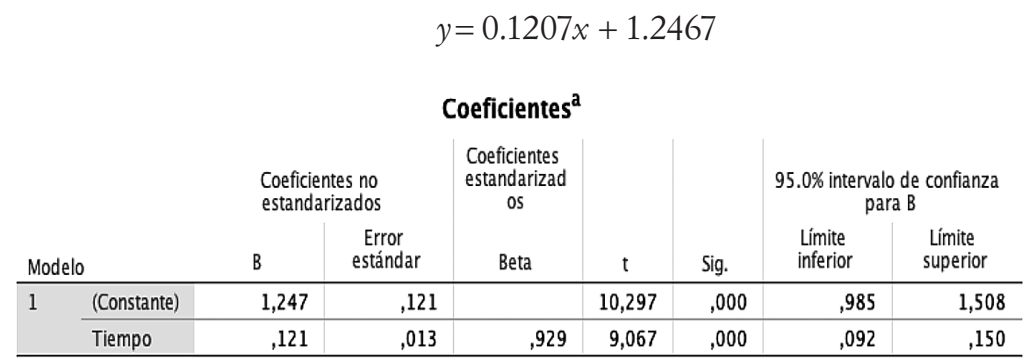

a. Variable dependiente: CitProGT

Figura 3. Análisis estadístico en SPSS para citaciones promedio de GT (regresión lineal)

La regresión arrojó un coeficiente $\mathrm{R}^{2}$ de 0.8635 (Véliz, 2011). La Figura 3 muestra sus pruebas estadísticas. Para ambos coeficientes, los p-values son menores a 0.05 , y los intervalos de confianza no pasan por 0 .

$\mathrm{Al}$ igualar ambas ecuaciones, se obtiene un valor de 3.88 para la variable tiempo. Es decir, las citaciones promedio por artículo fueron iguales entre 2006 y 2007, y en teoría ya no volverían a serlo. Sin embargo, también se generaron rectas de mejor ajuste polinómicas de segundo grado. A continuación se muestra la correspondiente a SEM:

$$
y=-0.0223 x^{2}+0.6548 x-0.4522
$$

La regresión arrojó un coeficiente $\mathrm{R}^{2}$ mucho más contundente de 0.9853 (Véliz, 2011). La Figura 4 muestra sus pruebas estadísticas, los intervalos de confianza no pasan por 0 .

Estimaciones de parámetro

\begin{tabular}{|c|c|c|c|c|}
\hline \multirow[b]{2}{*}{ Parámetro } & \multirow[b]{2}{*}{ Estimación } & \multirow[b]{2}{*}{$\begin{array}{l}\text { Error } \\
\text { estándar }\end{array}$} & \multicolumn{2}{|c|}{ Intervalo de confianza de 95} \\
\hline & & & $\begin{array}{l}\text { Límite } \\
\text { inferior }\end{array}$ & $\begin{array}{l}\text { Límite } \\
\text { superior }\end{array}$ \\
\hline A &,- 022 & ,003 &,- 028 &,- 016 \\
\hline B & ,655 &, 047 &, 553 &, 757 \\
\hline C &,- 452 & , 163 &,- 807 &,- 097 \\
\hline
\end{tabular}

Figura 4. Análisis estadístico en SPSS para citaciones promedio de SEM (regresión no lineal) 
La recta de mejor ajuste polinómica de segundo grado para GT es la siguiente:

$$
y=0.0077 x^{2}-0.0027 x+1.5966
$$

\section{Estimaciones de parámetro}

\begin{tabular}{|c|c|c|c|c|}
\hline \multirow[b]{2}{*}{ Parámetro } & \multirow[b]{2}{*}{ Estimación } & \multirow[b]{2}{*}{$\begin{array}{l}\text { Error } \\
\text { estándar }\end{array}$} & \multicolumn{2}{|c|}{ Intervalo de confianza de 95} \\
\hline & & & $\begin{array}{l}\text { Límite } \\
\text { inferior }\end{array}$ & $\begin{array}{l}\text { Límite } \\
\text { superior }\end{array}$ \\
\hline A &, 008 &, 003 & ,002 & 014 \\
\hline B &,- 003 &, 047 &,- 105 & 099 \\
\hline $\mathrm{C}$ & 1,597 &, 163 & 1,242 & 1,951 \\
\hline
\end{tabular}

Figura 5. Análisis estadístico en SPSS para citaciones promedio de GT (regresión no lineal)

Igual que para SEM, la regresión de GT obtuvo un mejor coeficiente $\mathrm{R}^{2}$, de 0.9154 (Véliz, 2011). La Figura 5 muestra sus pruebas estadísticas, y únicamente en el intervalo de confianza del parámetro B (el coeficiente que corresponde a la variable de grado uno), podría darse eventualmente que cobre el valor de 0 .

Al igualar las ecuaciones, se obtienen dos posibles respuestas: $3.7617 \mathrm{y}$ 18.155. Es decir, pasado el punto en el que ambas metodologías tuvieron un promedio igual de citaciones por artículo entre 2006 y 2007 (período 3), volverían a presentarlo en el año 2022 (período 18), para luego pasar GT a liderar el promedio.

\section{Discusión}

El volumen de producción y publicación intelectual vinculada a SEM tiene un comportamiento exponencial, mientras que el de GT es lineal e ínfimo. En función al comportamiento gráfico, la producción de artículos de SEM debería continuar en su tendencia, aunque los resultados llevan a cuestionar dichas publicaciones en términos de confianza (Cronin, 1984; Giffin, 1967; Nicolson, 2013), mostrada por la comunidad científica al citar o no dichas investigaciones. A pesar de que los artículos de GT son menores en volumen, presentan citaciones por artículo muy similares a las de SEM. Si el comportamiento de las citaciones promedio por investigación de ambos métodos se mantuviera, en 2022 podrían igualarse, lo que claramente determina que el volumen de investigaciones producidas no define el nivel de calidad ni de confianza depositados en dichos manuscritos a través de citaciones. 
Los hallazgos de la investigación, dado que el promedio de citaciones por artículo de SEM es mayor que el de GT, desmienten la $\mathrm{H}_{1}$ que planteaba su igualdad. Sin embargo, la $\mathrm{H}_{2}$ sí fue validada, ya que en el futuro se espera que las citaciones promedio para investigaciones de GT excedan a las de SEM. No obstante, más allá de las críticas recibidas a lo largo de los años (Brown, 1973; Bryman, 2004; Cutcliffe, 2000), los resultados evidencian que dicha metodología ha incrementado progresivamente la confianza recibida de parte de la comunidad científica. Aunque la investigación no apunta a encontrar las causas, según la literatura puede deberse a a) la rigurosidad metodológica (Gioia et al., 2013), b) la conveniencia de estudiar a los individuos más que sus decisiones (Graue, 2015), o c) la migración natural de ciertas disciplinas de estudio hacia lo cualitativo (Strandberg et al., 2018).

Los datos analizados en el artículo corresponden a toda la administración (Liu, Hong y Liu, 2012) según las categorías correspondientes en Scopus, lo que lleva a concluir preliminarmente que en efecto la comunidad científica cada vez deposita más su confianza (Cronin, 1984; Giffin, 1967; Nicolson, 2013) en la GT, aunque subdisciplinas como finanzas, economía, gestión, y emprendimiento presenten resultados contrarios entre sí (Cornelius y Persson, 2006). También es importante considerar que los resultados obtenidos en las regresiones no garantizan que ambas metodologías estudiadas vayan a igualar sus razones de citación en 2022. Antes de concluir ello, se debería tener en cuenta lo siguiente:

- La citación de un artículo se basa también en si el hallazgo es revolucionario. Si en los próximos años se publicara una investigación así, ya sea cualitativa o cuantitativa, sería muy citada y alteraría la tendencia actual de las citaciones promedio por artículo, por lo que el estudio debe ser replicado con cierta periodicidad para captar estos efectos.

- Existen fenómenos bibliométricos que pueden influenciar altamente la citación promedio por artículo. El prestigio de los autores o de las revistas (Di Vaio et al., 2012; Thornley et al., 2015), el acceso abierto a ellas (Cintra, Furnival y Milanez, 2018), entre otros, influyen en si una investigación es citada o no, por lo que estudiar revistas de cuartiles similares, autores con similar factor de impacto o segmentar por revistas libres y restringidas podría contribuir a mitigar estos efectos.

- Un fenómeno mucho más de fondo, y que tendría que ser explorado también, es si de forma sistemática se está migrando en la administración al estudio de la persona misma, más allá del resultado de sus decisiones (Graue, 2015), lo que inevitablemente tendría que ser atacado mediante una epistemología constructivista (Charmaz, 2000) y favorecería al paradigma cualitativo. 


\section{CONCLUSIONES Y RECOMENDACIONES}

Resulta relevante, para futuras investigaciones, considerar tres factores limitantes en el estudio. Primero, el prestigio que influye sobre las citaciones potenciales no fue aislado como factor exógeno (Thornley et al., 2015). Segundo, la GT sólo fue estudiada contra la SEM (Chión y Charles, 2016), excluyendo otras metodologías cuantitativas. Tercero, al procesar mayor cantidad de información mediante técnicas de Big Data E Analytics arrojaría resultados aún más contundentes, dividiendo a la administración en subdisciplinas que permitan entender si el comportamiento es sostenido para todas ellas, o si ciertos temas presentan tendencias particulares.

No obstante, existen oportunidades de investigación futura. El artículo no explora por qué se producen más artículos de SEM que de GT. Posiblemente su naturaleza constructivista hace que dicha metodología resulte más compleja, o menos preferida por falta de tiempo y recursos. Conocer esto ayudaría a impulsar investigaciones cualitativas brindándoles los recursos necesarios. Además, existe también la posibilidad de estudiar mayores horizontes de tiempo y volúmenes de información, sobre todo subdividiendo a la administración para una mejor comprensión de sus componentes disciplinarios. Sin embargo, lo que sí queda claro es que el estudio estadístico descriptivo sienta las bases para futuros modelos que puedan llevar a explicar las razones de por qué se está produciendo la migración de lo cuantitativo a lo cualitativo en las ciencias administrativas.

En conclusión, el paradigma cuantitativo fue arduamente defendido a través de las últimas décadas frente al fortalecimiento del cualitativo. Si bien ha sido probado que la GT genera teoría científicamente válida, el método cuantitativo, representado por la SEM, continúa liderando el índice de confianza medido en citaciones promedio por artículo. Esta visión de confianza, desde luego, se plantea como alternativa a la tradicionalmente utilizada de impacto, y cuenta con sustento teórico suficiente como para ser considerada como una medición válida de cuán contundente y confiable es una investigación. Sin embargo, el estudio bibliométrico, estadísticamente relevante y significativo en sus regresiones lineales y polinómicas, aunque no extrapolable a otras disciplinas y sin considerar las diferencias a nivel subdisciplinario, reveló que la diferencia de las citaciones promedio es ínfima comparada con el comportamiento de la producción intelectual total de ambos paradigmas. De esta forma, sólo se respalda la segunda hipótesis: en el futuro próximo es altamente probable que ambas razones se igualen y que la GT pase a liderar dicho indicador. Finalmente, es necesario continuar profundizando en cómo se comportan ambas metodologías, dados todos los fenómenos bibliométricos mencionados, y por qué. 


\section{REFERENCIAS}

Bengtsson, M. 2016. "How to plan and perform a qualitative study using concent analysis". NursingPlus Open 2: 8-14. https://doi.org/10.1016/j.npls.2016.01.001

Berk, J. B., C. R. Harvey y D. Hirshleifer. 2017. "How to write an effective referee report and improve the scientific review process". Journal of Economic Perspectives 31 (1): $231-244$ https://doi.org/10.1257/jep.31.1.231

Blalock, H. M. 1970. “An Introduction to Social Research”. Englewood Cliffs, NJ: Prentice Hall.

Brown, G. W. 1973. "Some thoughts on grounded theory”. Sociology 7 (1): 1-16. https://doi.org/10.1177/003803857300700101

Bryman, A. S. 2004. "Qualitative research on leadership: A critical but appreciative review". The Leadership Quarterly 15 (6): 729-769. https://doi.org/10.1016/j.leaqua.2004.09.007

Burton, B. 2007. "Qualitative research in finance - pedigree and renaissance". Studies in Economics and Finance 24 (1): 5-12. https://doi.org/10.1108/10867370710737355

Candia, R., B. Ortiz, L. G. de León, C. E. Carrasco y P. N. Gutiérrez. 2019. “Producción científica histórica de la Universidad Autónoma de Chihuahua: una revisión sistematizada". Investigación Bibliotecológica: archivonomía, bibliotecología e información 33 (79): 83-98. https://doi.org/10.22201/iibi.24488321xe.2019.79.57967

Cintra, P. R., A. C. Furnival y D. H. Milanez. 2018. “The impact of open access citation and social media on leading top Information Science journals". Investigación Bibliotecológica: archivonomía, bibliotecología e información 32 (77): 117-132. https://doi.org/10.22201/iibi.24488321xe.2018.77.57874

Charmaz, K. 2000. "Grounded theory and methodology: Objectivist and constructivist methods", en Handbook of Qualitative Research, 2a ed. Editado por N. K. Denzin y Y. S. Lincoln, 509-535. Londres: Sage Publications.

Charmaz, K. 2006. Constructing Grounded Theory. Thousand Oaks: Sage Publications.

Chión, S. y V. Charles. 2016. Analítica de datos para la modelación estructural. Lima: Pearson.

Cobo, M. J., M. A. Martínez, M. Gutiérrez-Salcedo, H. Fujita, y E. Herrera-Viedma. 2015. "25 years at Knowledge-Based Systems: A bibliometric analysis". Knowledge-Based Systems 80 (1): 3-13.

https://doi.org/10.1016/j.knosys.2014.12.035

Cornelius, B. y O. Persson. 2006. "Who's who in venture capital research". Technovation 26 (2): 142-150. https://doi.org/10.1016/j.technovation.2005.05.009

Creswell, J. 2007. Qualitative Inquiry E Research Design: Choosing among five approaches, $2^{a}$ ed. Thousand Oaks: Sage Publications.

Cronin, B. 1984. Citation Process: Role and Significance of Citations in Scientific Communication. Londres: Taylor Graham.

Cutcliffe, J. 2000. "Methodological issues in grounded theory". Journal of Advanced Nursing 31 (6): 1474-1484. 
Di Vaio, G., D. Waldenström y J. Weisdorf. 2012. "Citation success: Evidence from economic history journal publications”. Explorations in Economic History 49 (1): 92-104.

https://doi.org/10.1016/j.eeh.2011.10.002

Giffin, K. 1967. "The contribution of studies of source credibility to a theory of interpersonal trust in the communication process". Psychological Bulletin 68 (2): 104-120. http://dx.doi.org/10.1037/h0024833

Gioia, D. A., K. G. Corley y A. L. Hamilton. 2013. "Seeking Qualitative Rigor in Inductive Research: Notes on the Gioia Methodology”. Organizational Research Methods 16 (1): 15-31. https://doi.org/10.1177/1094428112452151

Glaser, B. y A. Strauss. 1967. The Discovery of Grounded Theory: strategies for qualitative research. New Jersey: Aldine Transaction.

Graue, C. 2015. "Qualitative data analysis". International Journal of Sales, Retailing and Marketing 4 (9): 2015.

Holland, J. 1998. "Private voluntary disclosure, financial intermediation and market efficiency". Journal of Business Finance and Accounting 25 (1/2): 29-68. https://doi.org/10.1111/1468-5957.00177

Jones, J. 2009. "Selection of Grounded Theory as an Appropriate Research Methodology for a Dissertation: One Student's Perspective”. The Grounded Theory Review 8 (2): 23-34.

Khresna, R., C. W. Hooy y Z. Ahmad. 2012. "Psychological factors on irrational financial decision making”. Humanomics 4 (2012): 236-257. https://doi.org/10.1108/08288661211277317

Liu, X., S. Hong y Y. Liu. 2012. "A Bibliometric Analysis of 20 Years of Globalization Research: 1990-2009”. Globalizations 9 (2): 195-210. https://doi.org/10.1080/14747731.2012.658256

Long, T. y M. Johnson. 2000. "Rigour, reliability and validity in qualitative research". Clinical Effectiveness in Nursing 4 (1): 30-37. https://doi.org/10.1054/cein.2000.0106

Martin, B. R. 2012. "The evolution of science policy and innovation studies". Research Policy 41 (7): 1219-1239. https://doi.org/10.1016/j.respol.2012.03.012

Mateos-Aparicio, G. 2011. "Los modelos de ecuaciones estructurales: una revisión histórica sobre sus orígenes y desarrollo", en Historia de la Probabilidad y la Estadística (V), editado por J. M. Riobóo y I. Riobóo, 289-302. Santiago de Compostela: Nino-Centro de Impresión Digital.

Merigó, J. M., A. Mas-Tur, N. Roig-Tierno y D. Ribeiro-Soriano. 2015. "A bibliometric overview of the Journal of Business Research between 1973 and 2014". Journal of Business Research 68 (12): 2645-2653. https://doi.org/10.1016/j.jbusres.2015.04.006

Merigó, J. M. y J. B. Yang. 2017. "A bibliometric analysis of operations research and management science". Omega 73 (December): 37-48. https://doi.org/10.1016/j.omega.2016.12.004

Moed, H. F., W. J. M. Burger, J. G. Frankfort y A. F. J. Van Raan. 1985. “The use of bibliometric data for the measurement of university research". Research Policy 14 (3): 131-149. https://doi.org/10.1016/0048-7333(85)90012-5 
Nicolson, D. 2013. "Taking epistemology seriously: 'truth, reason and justice' revisited”. The International Journal of Evidence \& Proof 17 (1): 1-46. https://doi.org/10.1350/ijep.2013.17.1.417

Pinegar, J. y L. Wilbricht. 1989. "What managers think of capital structure theory: a survey”. Financial Management 18 (4): 82-90. https://doi.org/10.2307/3665800

Poteshman, A. M. y V. Serbin. 2003. "Clearly irrational financial market behavior: Evidence from the early exercise of exchange traded stock options". The Journal of Finance 58 (1): 37-70. https://doi.org/10.1111/1540-6261.00518

Simkin, M. V. y V. P. Roychowdhury. 2003. "Read before you cite!" Complex Systems 14: 269-274. https://arxiv.org/pdf/cond-mat/0212043.pdf

Stern, P. 2007. "On solid ground: Essential properties for growing grounded theory", en The SAGE bandbook of grounded theory, editado por A. Bryant y K. Charmaz, 114-126. Londres: Sage Publications.

Strandberg, C., A. Nath, H. Hemmatdar y M. Jahwash. 2018. "Tourism research in the new millennium: a bibliometric review of literature in Tourism and Hospitality Research". Tourism and Hospitality Research 18 (3): 269-285. https://doi.org/10.1177/1467358416642010

Strauss, A. y J. Corbin. 1990. Basics of Qualitative Research: Grounded Theory Procedures and Techniques, 2a ed. Newbury Park: Sage Publications.

Sutcliffe, A. 2016. "Grounded theory: a method for practitioner research by educational psychologists". Educational and Child Psychology 33 (3): 44-54.

Tan, J. 2010. "Grounded theory in practice: issues and discussion for new qualitative researchers". Journal of Documentation 66 (1): 93-112. https://doi.org/10.1108/00220411011016380

Thornley, C., A. Watkinson, D. Nicholas, R. Volentine, H. R. Jamali, E. Herman, S. Allard, K. J. Levine y C. Tenopir. 2015. "The role of trust and authority in the citation bevahiour of researchers". Information Research 20 (3): 1-17.

Urquhart, C. y W. Fernández. 2013. "Using grounded theory method in information systems: the researcher as blank slate and other myths". Journal of Information Technology 28: 224-236. https://doi.org/10.1057/jit.2012.34

Verbeek, A., K. Debackere, M. Luwel y E. Zimmermann. 2002. "Measuring progress and evolution in science and technology - I: The multiple uses of bibliometric indicators". International Journal of Management Reviews 4 (2): 179-211. https://doi.org/10.1111/1468-2370.00083

Viktorovna, B., G. Pavlovna, y Z. Mokhailovna. 2018. "Decision-making at the different levels of rationality: subjects' cognitive, neural and psico-dynamic characteristics". International Journal of Cognitive Research, Science, Engineering and Education 6 (1): 39-44. https://doi.org/10.5937/ijcrsee1801039B

Véliz, C. 2011. Estadística para la administración y los negocios. Ciudad de México: Pearson. 
Zupic, I. y T. Čater. 2015. "Bibliometric Methods in Management and Organization”. Organizational Research Methods 18 (3): 429-472.

https://doi.org/10.1177/1094428114562629

Para citar este texto:

Arana Barbier, Pablo José. 2020. "Situación de la confianza en la teoría fundamentada versus la modelación de ecuaciones estructurales: un estudio bibliométrico comparativo para la administración". Investigación Bibliotecológica: archivonomía, bibliotecología e información 34 (83): 37-53.

http://dx.doi.org/10.22201/iibi.24488321xe.2020.83.58111 